Cuadernos de Filología Clásica. Estudios Latinos ISSN: 1131-9062

http://dx.doi.org/10.5209/CFCL.57806

\title{
Gramática modista versus gramática sanctiana: dos concepciones de análisis lingüístico contrapuestas ${ }^{1}$
}

\author{
Victoria Manzano Ventura²
}

Recibido: 13 de junio de 2017 / Aceptado: 4 de octubre de 2017

Resumen. La teoría gramatical modista siempre se ha considerado un precedente claro de la teoría gramatical sanctiana. Es evidente que ambas teorías comparten un mismo objetivo de universalidad lingüística. Sin embargo, ese mismo objetivo teórico se intenta alcanzar en ambas doctrinas mediante patrones metodológicos muy diferentes. Y es que la gramática bajomedieval modista y la de Francisco Sánchez de las Brozas (Franciscus Sanctius Brocensis) están separadas, como analizamos en nuestro estudio, por una contrapuesta concepción de análisis lingüístico respecto al discurso figurado que determina la exclusión por sistema del mismo en el modismo frente a su estudio racional y sistemático en la gramática de Sanctius. Y tal contrapuesta concepción de análisis lingüístico no se ha destacado ni enfatizado lo suficiente a pesar de que es clave tanto para interpretar correctamente estos dos decisivos y trascendentales sistemas gramaticales como para precisar las aportaciones esenciales de cada uno a la lingüística de Occidente.

Palabras clave: Gramática modista; gramática sanctiana; discrepancia en el análisis lingüístico; discurso figurado.

\section{[en] Modistic grammar versus Sanctian grammar: two conflicting conceptions of linguistic analysis}

Abstract. The grammatical theory of the modistae has always been considered to be a clear precedent of the grammatical theory of Sanctius. It is evident that both theories share the same objective of linguistic universality. However, both doctrines seek to achieve that single theoretical objective through very different methodological patterns. In fact, the grammar of the modistae, from the late Middle Ages, and that of Franciscus Sanctius Brocensis (Francisco Sánchez de las Brozas) are separated, as we analyse in our study, by a conflicting conception of linguistic analysis with respect to the figurative discourse, which determines the systematic exclusion of the same in Modistic grammar compared to its rational and systematic study in Sanctian grammar. And this conflicting conception of linguistic analysis has not been highlighted nor emphasised sufficiently, despite being key to properly interpreting these two decisive and transcendental grammatical systems, each providing essential contributions to Western linguistics.

Keywords: Grammar of the modistae; grammar of Sanctius; discrepancy in linguistic analysis; figurative discourse.

\footnotetext{
1 Este trabajo se enmarca en el Grupo de Investigación LAPAR (HUM 002) dirigido por el admirado Prof. D. Eustaquio Sánchez Salor y financiado por los fondos FEDER a través del IV Plan Regional de I+D+I del Gobierno de Extremadura.

2 Universidad de Extremadura (España) victoriamv@unex.es.
} 
Sumario: 1. Introducción. 2. La teoría modista: una gramática profunda de enunciados correctos y estándares. 3. El discurso figurado en la gramática premodista: los antecedentes del posterior enfoque modista. 4. La gramática sanctiana: el estudio racional y sistemático del discurso figurado. 5. Conclusión. 6. Referencias bibliográficas.

Cómo citar: Manzano Ventura, V., «Gramática modista versus gramática sanctiana: dos concepciones de análisis lingüístico contrapuestas», Cuad. Filol. Clás. Estud. Lat. 37.2 (2017), 283-301.

\section{Introducción}

La gramática modista y la sanctiana son, sin duda, las dos teorías lingüísticas -y el término se les puede aplicar con total propiedad- más relevantes y conocidas del período medieval y renacentista de manera respectiva.

Afortunadamente, desde hace aproximadamente unos cincuenta años, gracias a la posición central del generativismo y al avance espectacular y decisivo de la historiografía lingüística, ambas teorías gramaticales han sido estudiadas, analizadas y revalorizadas en gran medida ${ }^{3}$.

De este modo, desde que en 1966 Noam Chomsky publicara su célebre y conocida Lingüistica cartesiana, la vinculación conceptual y teórica entre la gramática generativa y la Gramática general y razonada del siglo XVII quedó fijada ${ }^{4}$. Apenas tres años más tarde, Robin Lakoff, con mejor y más amplia perspectiva, estableció una prefiguración de las tesis generativistas en la Minerua sanctiana, fuente indiscutible, a su vez, de la gramática de Port-Royal ${ }^{5}$. Y, a partir de ahí, han sido muchos los estudios que han sugerido y determinado una vinculación y una conexión entre la gramática modista, la gramática sanctiana y ciertos planteamientos generativistas ${ }^{6}$.

Para una aproximación general a los rasgos definitorios de la teoría modista destacan junto a obras ya clásicas como las de Bursill-Hall (1971), Pinborg (1967), Rosier (1983a) o Covington (1984), otras más modernas como las de Kelly (2002) o Beuerle (2007). Asimismo, para una exposición completa de la doctrina sanctiana en sus diferentes aspectos teóricos y metodológicos, afortunadamente, pueden consultarse en la actualidad múltiples estudios y trabajos, entre otros, Clerico (1977, 125-143), Breva Claramonte (1983), Codoñer Merino (1988, 177-182), Stéfanini (1994a, 97-107), Sánchez Salor (2000, 13- 42; 2002, 442-450, 493-518, 565-587; 2003, 61-80), Mañas Núñez (2010, 209-235).

4 Chomsky (1966, 31-51). Sin embargo, en esta obra Noam Chomsky atribuye todo el mérito del inicio de la tradición logicista en la lingüística de Occidente a la gramática de Port-Royal, relegando el fundamental papel desempeñado al respecto por la Minerua de Sanctius a una brevísima nota, (Chomsky 1996, 97 n.67): «Apart from its Cartesian origins, the Port-Royal theory of language, with its distinction between deep and surface structure, can be traced to scholastic and renaissance grammar; in particular, to the theory of ellipsis and 'ideal types' that reached its fullest development in Sanctius' Minerua (1587)». Y más desconcertante resulta aún la valoración por parte del lingüista estadounidense en su obra Language and mind (1968) donde asimila la teoría de la elipsis de Sánchez a un mero procedimiento para la interpretación de textos, sin considerar ni valorar la importancia decisiva que el análisis en dos niveles de descripción gramatical juega en la Minerua sanctiana y también en la evolución posterior de la teoría gramatical europea, uid. Chomsky $(1968,16)$.

5 Lakoff $(1969,356)$. Cf. Lakoff (1973).

6 La vinculación en diferentes aspectos y planteamientos entre la gramática modista, la Minerua de Sanctius y ciertas tesis chomskianas figura en múltiple estudios, obras y trabajos del campo de la historiografía lingüística publicados en los últimos cuarenta años. 
Evidentemente, nunca se podrá alabar ni aplaudir lo suficiente este renovado interés por el conocimiento de la tradición lingüística occidental a lo largo de la historia -desde su mismo origen hasta nuestros días-, pues ha permitido rescatar del olvido y valorar en su justa medida muchas escuelas, corrientes y autores que permanecían sepultados por el paso del tiempo y minusvalorados por el desconocimiento de sus -a veces, muy meritorias- concepciones lingüísticas.

Sin embargo, siempre que se establece y determina una vinculación o conexión entre escuelas y corrientes lingüísticas separadas por distancias abusivas en el tiempo, hay que hacerlo con suma precisión, rigor y también precaución, pues, de lo contrario, por el afán de similitud, se corre el riesgo de caer en una falsa y simplista uniformización doctrinal, perdiendo incluso, a veces, de vista la esencia misma de las dos teorías lingüísticas comparadas. Pues bien, este modo de proceder se aprecia con no poca frecuencia en obras, estudios y manuales -siempre hablando en un sentido general- en los que se alude a la gramática modista como un antecedente claro de la teoría gramatical sanctiana sin mayor especificación ni aclaración doctrinal, es decir, como si ambas corrientes lingüísticas compartieran idénticos patrones doctrinales, lo cual está muy lejos de la realidad. $\mathrm{Y}$ es que, aunque los modistae a finales del período bajomedieval y el Brocense en la segunda mitad del XVI persigan un mismo objetivo de universalidad y cientificidad lingüística, lo hacen desde posiciones metodológicas no ya diferentes, sino opuestas completa y diametralmente. $Y$ este es un aspecto de gran importancia al que ni se le presta ni se la ha prestado la atención suficiente a pesar de que es fundamental para interpretar correctamente la originalidad y el alcance lingüístico de ambas teorías gramaticales.

Veamos brevemente, por tanto, lo marcado y significativo de estas irreconciliables diferencias, examinando, en primer lugar, los grandes temas y principios teóricos y metodológicos del modismo que, aun hoy en día, por su complejidad e indefinición, se prestan a equívoco y confusión?

\section{La teoría modista: una gramática profunda de enunciados correctos y estándares}

Es hacia el último tercio del siglo XIII en el entorno averroísta de la Universidad de París donde se componen los primeros tratados de modis significandi, y ello marcaría el surgimiento definitivo de la teoría modista ${ }^{8}$. O dicho en términos más significativos, surgía, por vez primera en la historia de la lingüística, una teoría general del lenguaje de base universal y científica en lo que puede ser considerado una auténtica revolución teórica y metodológica. Y es que los modistae, para la configuración de su gramática universal, dejan atrás una metodología de análisis con más de mil cuatro-

\footnotetext{
La gramática modista, por su elevada complejidad teórica, falta de claridad en determinados aspectos básicos y medulares, y ausencia de definiciones de los constituyentes esenciales del sistema (primum-secundum, dependens-terminans, etc.), no resulta de fácil interpretación ni análisis para los especialistas y estudiosos modernos. Y ello ha determinado interpretaciones confusas y divergentes sobre la presencia, operatividad y alcance de ciertas nociones lingüísticas clave en la teoría sintáctica modista: elipsis, estructura profunda/ estructura superficial, concordancia/régimen, etc.

8 El De modis significandi de Martín de Dacia es considerado el primer tratado propiamente modista conservado. Su fecha de composición suele fijarse, a partir de diferentes datos tanto internos como externos, en torno a 1270.
} 
cientos años de vigencia, es decir, rompen con la gramática esencialmente práctica y normativa que había imperado hasta entonces para desarrollar una nueva y revolucionaria teoría de análisis lingüístico de carácter general y alcance universalista9

En tan innovadora teoría la pieza básica y medular es el conocido concepto de modus significandi que permite designar una misma entidad con un significado básico (significatio) de maneras diferentes en virtud de un significado adicional (consignificatio), por ejemplo, como un suceso estático, una acción en proceso o una cualidad ('estudio', 'estudiar', 'estudioso'....) ${ }^{10}$. Dicha característica propiedad designativa (consignificatio) se explica y se justifica a partir de la analogía simétrica y sin precedentes que los modistae establecen entre los planos de la realidad, del entendimiento y del lenguaje, premisa indispensable para establecer la conclusión universalista de su teoría: si la estructura de la realidad es la misma para todos, entonces la gramática también ha de serlo ${ }^{11}$. La gramática se convierte así para los modistae en el estudio de los diferentes modi significandi característicos de cada parte de la oración.

Y sobre este nuevo marco conceptual de base ontológica y cognitiva los modistae configuraron su elaborada teoría sintáctica. Al respecto, la crítica, en general, sostiene que la sintaxis es el fin último de la teoría modista y también su apartado más relevante. Tal afirmación, sin embargo, ha de asumirse con muchas precauciones. Lo único cierto es que el apartado dedicado a la teoría sintáctica se ha perdido en la mayoría de tratados y manuales. De hecho, solo en dos de las obras editadas, la de Martín de Dacia (ca. 1270) $)^{12}$ y Tomás de Erfurt (ca. 1300) ${ }^{13}$, es posible encontrar completa la sección de sintaxis (diasynthetica). Las Quaestiones super Priscianum Minorem de Rodolfo Brito (ca. 1300) ${ }^{14}$ pueden añadirse a este reducido listado, aunque solo sensu lato, pues la obra de Brito no constituye una gramática al uso, sino una recapitulación y discusión teórica sobre diferentes planteamientos problemáticos de carácter sintáctico. Conservamos, asimismo, algunas secciones de sintaxis de la Summa modorum significandi de Siger de Courtrai (ca. 1320) ${ }^{15}$, pero en lo sustancial su tratamiento de la sintaxis también se ha perdido.

Sea como fuere, y a pesar de estas limitaciones, los textos conservados -por su profundización teórica- ofrecen un excelente testimonio sobre el modelo sintáctico modista. Dicho modelo está conformado, tanto en los primeros años de desarrollo del modismo como en la última etapa de mayor madurez teórica, por tres etapas sucesivas o passiones grammaticales según la nueva y precisa terminología modista

$9 \quad$ El paso de ars a scientia supone un salto cualitativo de fundamental importancia en la teoría gramatical de la Edad Media, $c f$. Rosier (1983, 23ss.)

10 El término modus significandi fue adoptado, según Thurot (1964, 50), de Boecio. Aunque el concepto se registra en la Baja Edad Media con anterioridad a la configuración del género de modis significandi en autores como Abelardo, Bernard de Chartres o Pedro Helías, lo cierto es que solo en los tratados modistas aparece como concepto clave y vertebrador de la teoría gramatical. Sobre la problemática conceptual que este término medular plantea en los tractatus y comentarios de modis significandi, cf. Roos (1961, 8), Pinborg - Roos (1969, 62-86).

1 La relación simétrica entre realidad, entendimiento y lenguaje es el fundamento sobre el que los modistae levantan su teoría de la gramática, una gramática que, al tener una base ontológica y cognitiva igual para todos, aspira a ser universal, $c f$. Bursill-Hall (1971, 13, 35 y 332-336; 1976, 165), Kelly (1977, 123), Rosier (1981, 61), Stéfanini (1994b, 63).

12 Vid. Roos (1961, 85-118).

13 Vid. Bursill-Hall (1972, 272-321).

14 Vid. Enders - Pinborg (1980, 89-436).

15 Vid. Pinborg (1977, 1-33). 
derivada de la física de Aristóteles ${ }^{16}$ : constructio o unión de dos palabras, congruitas o formación correcta de la construcciones, y perfectio o completud de un enuncia$\mathrm{do}^{17}$. Estas tres passiones se superponen entre sí en un orden ascendente. O dicho de otro modo, cada nivel presupone al que le precede (manifiestum est quod constructio prior est congruitate et congruitas perfectione $)^{18}$. Todo ello en busca de la finalidad última de la oratio perfecta y, por ende, de la gramática según la consideración modista: comunicar un pensamiento completo en la mente del oyente, es decir, lograr la función comunicativa ${ }^{19}$.

Pero este cuadro general teórico y metodológico del modismo encierra en su misma formulación el gran problema y también la gran contradicción y paradoja de la teoría sintáctica modista que la crítica, centrada de manera prioritaria en subrayar las similitudes y paralelismos -que, evidentemente los hay- entre la gramática modista, la sanctiana y la lingüística moderna, no enfatiza o pasa por alto a pesar de su fundamental importancia. Y es que, desde el momento en que en la sistematización teórica modista se establece como principio básico y medular que el nivel de la perfectio presupone al de la congruitas ${ }^{20}$, solo es posible alcanzar esa anhelada función comunicativa de un modo muy limitado, el que atañe única y exclusivamente a los enunciados correctos, canónicos y estándares, nada más. El resto de enunciados de una lengua que, a pesar de su impropiedad -o fingida impropiedad gramatical - y carácter figurado, tienen pleno éxito y sentido en la realidad comunicativa se rechazan y se excluyen por sistema de la teoría sintáctica modista ${ }^{21}$.

Sin embargo, la fácil y simplista solución adoptada por el modismo de apartar de la gramática, sin más, los enunciados figurados al no poder explicarlos, evidentemente, planteaba múltiples dificultades y problemáticas de aplicación. Es preciso, por tanto, rastrear y revisar las alusiones a esta cuestión presentes desde el comienzo hasta el final del género en los tratados De modis significandi conservados. De este modo, será posible determinar con precisión cuál es la relevancia y el tratamiento teórico que la exclusión de las construcciones figuradas alcanza en el sistema gramatical modista.

Por lo que a la primera generación de gramáticos modistas se refiere (1270-1285) y, más concretamente, al tratado de Martín de Dacia -aunque muy completo y exhaustivo, único texto sintáctico conservado de este primer período-, sorprende y decepcio-

16 Cf. Beuerle (2010, 276): Tatsächlich verweist der Begriff passio auf die der mittelalterlichen und modistischen Konzeption der Syntax zugrunde liegende Vorstellung, dass die Formierung von Wörtern zur Rede der Bewegung von Dingen im Raum entspricht. Die einzelnen partes orationis sind damit Dinge, die eine Bewegung, erleiden bzw. erfahren ( pati), indem sie durch bestimmte Abhängigkeiten, die sich wiederum aus ihren verschiedenen Bezeichnungsweisen ergeben, zueinander hingezogen oder voneinander getrennt warden.

17 Vid. Roos (1961, 85-115) y Bursill-Hall (1972, 272-320).

$18 \operatorname{Vid}$. Roos $(1961,87)$.

19 Roos (1961, 113), Bursill-Hall (1972, 316).

20 Roos $(1961,115)$, Bursill-Hall $(1972,318)$.

${ }^{21}$ Como se sabe, toda construcción figurada (figurativa constructio) se caracteriza por la presencia de una impropiedad en la ordenación habitual de los elementos de una construcción; pero se trata de una impropiedad que, a diferencia del uitium, siempre se produce y se justifica por una razón (ratio excusans). De este modo, el discurso figurado no rompe ni la claridad ni el sentido de la comunicación. Por ejemplo, en la construcción figurada populus currunt la impropiedad toma la forma de falta de correspondencia entre el número singular del sujeto (populus) y el plural del verbo (currunt), pero se justifica por la característica de nombre colectivo del sustantivo populus que, a pesar de estar en singular, hace referencia a un grupo de personas. Por ello, aunque en este tipo de construcciones la corrección gramatical está rota, el sentido se mantiene y la comunicación se logra de manera exitosa. 
na al mismo tiempo no encontrar ningún tipo de argumentación teórica justificativa para una cuestión tan esencial como la no aceptación del discurso figurado.

De este modo, en el tratamiento por parte del filósofo de Dacia de las constructiones intransitivae a parte post se buscará en vano una explicación sobre la elipsis del suppositum implícito en las construcciones con verbo vocativo: (ego) vocor Iohannes (Roos 1961, 105). Y más significativo resulta aún el vacío explicativo en el apartado que Martín dedica al análisis de la perfectio gramatical donde critica expresamente a quienes defienden y sostienen que en las construcciones figuradas el nivel de la perfectio no presupone al de la congruitas: perfectio non praesupponat congruitatem (Roos 1961, 112). La crítica va dirigida, por tanto -con todo lo que ello conlleva-, contra quienes rechazan y no admiten el principio básico y medular de corrección lingüística modista por lo que a las tres passiones grammaticales se refiere. Sin embargo, y contrariamente a lo que cabía esperar, Martín se limita a negar la opinión en cuestión y nada más (Roos 1961, 112-113). No hay ninguna razón explicativa ni tampoco ningún argumento probatorio. El vacío extraña en gran medida desde una perspectiva actual: la toma de postura más relevante y controvertida de la gramática modista no se apoya con ningún tipo de argumentación teórica.

En la segunda generación de gramáticos modistas (1285-1310) la complejidad y precisión de la doctrina alcanzan su culmen. En sintaxis el marco teórico de Martín se mantiene en términos generales, pero se revisa y perfecciona en algunas cuestiones para lograr un sistema lo más coherente posible.

En esta dinámica de mayor exhaustividad y rigor doctrinal se insertan algunas precisiones que atañen al análisis del discurso figurado en el modismo. Pero se trata, en cualquier caso, de precisiones sumamente parcelarias que no abordan la problemática en su conjunto ni de una manera sistemática. Estas innovaciones en la teoría son introducidas por quienes son considerados, sin duda, los filósofos más representativos e influyentes de esta última etapa de desarrollo modista: Rodolfo Brito y Tomás de Erfurt.

En efecto, Rodolfo Brito en su obra principal, Quaestiones super Priscianum Minorem $^{22}$, establece en el nivel de la congruitas gramatical una importantísima distinción -no presente en la teoría de Martín- entre congruitas secundum sensum y congruitas secundum intellectum. Especial relevancia e interés merece esta última consideración, pues para el gramático bretón la congruitas secundum intellectum posibilita la gramaticalidad de enunciados con el sujeto elíptico ${ }^{23}$. Y en esta misma línea de innovación teórica, y dada la superposición ascendente -ya señalada- de las tres passiones sermonis, Rodolfo introduce idéntica consideración también en su análisis de la perfectio al distinguir entre perfectio secundum sensum y perfectio secundum intellectum. Con la perfectio secundum intellectum Rodolfo hace referencia a los enunciados completos en virtud del entendimiento ${ }^{24}$.

Apenas unos años más tarde, Tomás de Erfurt asume plenamente la novedosa distinción establecida por el gramático bretón ${ }^{25}$ y la incorpora -otorgándole plena

22 En su obra culmen Rodolfo Brito aborda diferentes aspectos controvertidos y discusiones en torno a la teoría sintáctica modista. Sigue para su composición en gran parte la estructura del Priscianus Minor, si bien se añaden una serie de quaestiones introductorias dedicadas a los problemas universales de la gramática, $c f$. Enders - Pinborg (1980, 89-96).

23 Enders - Pinborg (1980, 341). Cf., asimismo, Enders - Pinborg (1980, 180-181).

24 Enders - Pinborg (1980, 180).

25 Bursill-Hall $(1972,312,314,318)$. Cf. Covington $(1984,70)$. 
validez teórica- al manual modista de referencia y de mayor influencia y difusión, su Grammatica Speculativa fechada en torno al $1300^{26}$.

Por supuesto, la distinción ad sensum / ad intellectum, presente ya en las Quaestiones de Rodolfo Brito y heredada por Tomás de Erfurt, supone una reflexión lingüística sugerente y también interesante respecto a la primera generación de gramáticos modistas. Pero tal consideración no significa, como a menudo se ha pretendido, que en la gramática modista se opere ya con un doble nivel del lenguaje. Y ello se demuestra con total evidencia a partir de dos razones: primera, y en un nivel general de formulación teórica, porque la distinción entre gramática superficial y real no tiene cabida -de ningún modo- en la elaborada sistematización modista que solo opera, por definición, con una gramática de enunciados correctos y estándares; y segunda, y en un plano concreto de aplicación, porque en la gramática de modis significandi la referencia a enunciados implícitos se limita única y exclusivamente a la elipsis del sujeto pronominal sin profundizar ni ir más allá en el análisis ${ }^{27}$.

Lo cierto es que las construcciones con el sujeto elidido -lego- constituían una problemática de alcance y un desafío para el modismo. Y es que, debido a su frecuencia de uso, este tipo de construcciones no podían quedar excluidas sin más -como el resto de enunciados anómalos- de la gramática. Pero, al mismo tiempo, ello implicaba dar entrada en el exhaustivo y canónico sistema de modis significandi a una serie de enunciados no completos y, por tanto -según la consideración modista-, no correctos desde un punto de vista lingüístico. La contradicción resultaba evidente; la aporía, insalvable.

$\mathrm{Y}$ es la necesidad de ofrecer algún tipo de explicación a una incoherencia tan flagrante en el sistema lo que, a nuestro juicio, dicta y determina la innovación que Rodolfo Brito y Tomás de Erfurt proponen en los niveles de perfectio y congruitas. No olvidemos que en la segunda etapa de desarrollo modista (ca. 1300-1310) la teoría alcanza su máximo apogeo precisamente en función del esfuerzo realizado por los autores de este segundo período para perfeccionar el sistema heredado en sus puntos más polémicos.

El problema es que la respuesta aportada por Brito y Tomás de Erfurt -aunque interesante desde un punto de vista lingüístico-, por su imprecisión y carácter reduccionista, al aplicarse solo a los enunciados con sujetos sobreentendidos, está muy lejos de solucionar la problemática del discurso figurado en el modismo. Muy al contrario, la distinción ad sensum / ad intellectum plantea, de manera inevitable, nuevos interrogantes sin respuestas a nivel de concepción teórica general y pone aún más en evidencia las contradicciones e inconsecuencias de un sistema que es preso del principio que lo sustenta: la exclusión del discurso figurado.

En efecto, la distinción ad intellectum que tanto Brito como Tomás de Erfurt proponen parece plantear la existencia de una estructura aún más profunda en el sistema; pero esa estructura aún más profunda ni se explica ni se define ni se delimita $\mathrm{y}$, además, se aplica, de forma unilateral, solo y exclusivamente a los enunciados con

26 La Grammatica Speculativa de Tomás de Erfurt reemplazó rápidamente, a inicios del XIV, a la de Martín de Dacia como manual de referencia y, además, tuvo una presencia muy significativa en la escuela de Centroeuropa en este período, $c f$. Pinborg $(1967,131)$.

27 Vid. Bursill-Hall (1972, 312): [...] contingit in uerbis primae et secundae personae, in quibus intelligitur suppositum, y Bursill-Hall (1972, 318). Asimismo, cf. Enders - Pinborg (1980, 198, 215-218). 
el sujeto elidido ${ }^{28}$. Todo lo cual añade aún mayor confusión y equívoco a la teoría lingüística modista en relación al discurso figurado.

Con todo $-y$ es fundamental subrayar esta idea-, incluso en la explicación de los enunciados con el sujeto sobreentendido, los modistae se aferran y no renuncian a los principios básicos y definitorios de su teoría. Y dicha teoría implica, como se ha señalado, el rechazo per se de un doble nivel de descripción lingüística y también, por ende, de la figura de construcción. Así, para los modistae entre dos enunciados como lego y ego lego hay una equivalencia total y absoluta, es decir, no hay entre ellos la más mínima diferencia de significado y el pronombre personal ego no adquiere, por tanto, valor de oposición alguna. La única diferencia estriba en que en ego lego la construcción es ad sensum -los dos constituyentes están expresos-, mientras que en lego la construcción es ad intellectum - uno de los constituyentes es percibido por el intelecto-; pero el valor semántico es, según la consideración modista, idéntico en ambos $\operatorname{casos}^{29}$ :

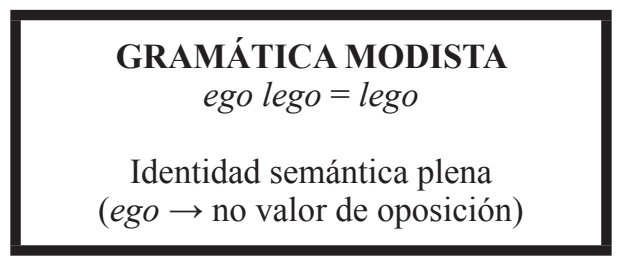

En definitiva, la revisión, a través de los distintos tratados y manuales de modis significandi conservados, de una cuestión tan esencial en el modismo como la exclusión del discurso figurado pone sorprendentemente de manifiesto la ausencia de cualquier tipo de reflexión teórica al respecto. Es como si los modistae dieran por asumida, sin más, dicha exclusión en su sistema y no sintieran, por tanto, la necesidad de explicarla y justificarla con razones y argumentos probatorios. Sin embargo, resulta sumamente intrigante desde nuestra perspectiva que en un sistema tan elaborado y preciso como el modista el porqué de la no aceptación del discurso figurado -principio lingüístico de fundamental alcance- no tenga respuesta.

Con todo, y a nuestro juicio, tan sorprendente vacío teórico tiene una explicación, solo que esa explicación no se halla en una perspectiva lingüística sincrónica, sino diacrónica. Y es que no es la etapa modista en sí, sino en la inmediatamente anterior donde hay que situar -según nuestra opinión- el tratamiento, el debate y la subsiguiente solución que en el último cuarto del siglo adoptarán los modistae respecto a la problemática del discurso figurado y para la que ellos ya no ofrecerán explicación teórica alguna por considerarla conocida y asumida a partir de la generación de gramáticos previa.

En efecto, la radical y relevante toma de postura del modismo en este sentido no es ni tan casual ni tan sorprendente como en principio pudiera parecer, pues, en rea-

28 Cf. Bursill-Hall $(1971,332)$ : «It is reasonable to say that the Modistae, by the very nature of their theoretical criteria, were concerned with deep structure almost to the total exclusion of surface grammar». Sin embargo, el papel de la «gramática superficial» en el modismo, por la complejidad y falta de precisión del elaborado sistema gramatical modista, no siempre ha sido bien interpretado por estudiosos y especialistas.

29 Rodolfo Brito es el gramático modista que desarrolla con mayor detenimiento y exhaustividad teórica la explicación de los enunciados con el sujeto sobreentendido, uid. Enders - Pinborg (1980, 215-218). Cf. Rosier (1983b, 34). 
lidad, responde a la evolución de las ideas lingüísticas en la primera mitad del siglo XIII, etapa gramatical, en general, poco conocida, estudiada y valorada, que recibe la vaga denominación de período premodista y que abarca, aproximadamente, del 1200 al $1260^{30}$. Ensombrecidas y eclipsadas en gran medida por los logros y hallazgos del modismo, las obras premodistas contienen, no obstante -a pesar de que, a menudo, no se les presta la atención que merecen- muchas de las claves interpretativas de la posterior sistematización modista. Entre ellas, a nuestro juicio, la que atañe a la no aceptabilidad de los enunciados figurados. Es obligado, por tanto, revisar y analizar brevemente cómo se afronta y dirime en la primera mitad del siglo la compleja problemática sobre la legitimidad o no del discurso figurado, pues en dicha controversia se hallan las coordenadas lingüísticas que explican y justifican el rompedor posicionamiento del modismo en este sentido.

\section{El discurso figurado en la gramática premodista: los antecedentes del posterior enfoque modista}

Lo cierto es que mucho antes de la primera mitad del siglo XIII, ya desde el inicio mismo de la reflexión sintáctica latina, es decir, en las propias Institutiones de Prisciano (s. VI d.C), es posible hallar apuntes más que interesantes sobre la contraposición entre gramaticalidad e inteligibilidad de un enunciado:

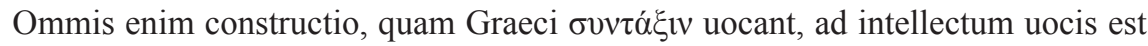
reddenda. Itaque per diuersas figuras uariare solent auctores in constructione accidentia [...], quae, quamuis quantum ad ipsas dictiones incongrue disposita esse uideantur, tamen ratione sensus rectissime ordinata esse iudicantur (Hertz 1961, 201).

Así pues, toda construcción, denominada por los griegos 'sintaxis', debe atender siempre a la significación de las formas. Ahora bien, los autores, mediante figuras diversas, suelen variar los accidentes en la construcción [...] de manera que, aunque la relación parezca incorrecta en cuanto a las formas concretas, sin embargo, racionalmente, se mostrará correcta (Harto Trujillo 2015, 214).

Pero las significativas palabras del de Cesarea no recibieron atención ni consideración y permanecieron durante muchos siglos en el olvido. Y es que no es hasta mediados del siglo XII cuando el comentarista de Prisciano más conocido e influyente del período bajomedieval, Pedro Helías, da un paso absolutamente decisivo en este sentido al distinguir, por vez primera, en la gramática de Occidente entre 'congruencia morfosintáctica' (congrua uoce) y 'congruencia semántica' (congrua sensu):

Constructio est congrua dictionum ordinatio. 'Congrua' intelligendum est tam uoce quam sensu. Tunc est ordinatio dictionum congrua uoce quando uoces secundum sua accidentia congrue sibi coniunguntur $[\ldots]$ Congrua uero sensu est

30 De hecho, contrasta la ausencia, en general, de monografías dedicadas al análisis del llamado «premodismo» con la multitud de libros y estudios que centran su atención e interés, de manera específica, en la teoría modista. 
ordinatio dictionum quando ex dictionibus predicto modo ordinatis habet auditor quid rationabiliter intelligat siue uerum sit siue falsum (Reilly 1993, 832) ${ }^{31}$.

La oración es una ordenación congruente de palabras. 'Congruente' debe entenderse tanto en la forma como en el sentido. De este modo, hay una ordenación congruente de palabras en cuanto a la forma cuando las palabras se unen de manera coherente según sus accidentes [...] A su vez, hay una ordenación congruente de palabras en cuanto al sentido cuando, a partir de las palabras ordenadas según lo dicho, el oyente es capaz de comprender racionalmente si lo expresado es verdadero o falso ${ }^{32}$.

Sin embargo, la fundamental distinción de Helías tampoco alcanzó importancia significativa en el desarrollo gramatical y sintáctico de la Europa medieval de mediados y finales del $\mathrm{XII}^{33}$, pues es solo en la primera mitad del XIII cuando la discusión y el debate sobre la aceptación o no de las construcciones figuradas pasa a primer plano de la gramática y alcanza un fundamental e inusitado protagonismo y alcance. Las razones de esta novedosa relevancia están más que claras.

Son los gramáticos y teóricos de principios y de mediados del siglo XIII los que -aunque sin la explicación doctrinal y filosófica del modismo posterior- definitivamente cambian el rumbo hacia una reorientación científica y universal de la gramática. $\mathrm{Y}$ en ese proceso de elevación de la gramática a la categoría de ciencia resultaba imprescindible - por razones obvias- operar con el mayor número posible de unidades sistematizables y organizadas. Pero las construcciones figuradas, por definición, quedaban al margen de cualquier intento de sistematización y uniformización lingüísticas.

Para solventar la dificultad, los eruditos y tratadistas de la primera mitad del XIII desarrollan su teoría del doble intellectus. La meritoria teoría consiste -en términos generales y sin entrar en particularismos- en la distinción de un intellectus primus cuyo significado lo configuran las formas lingüísticas del discurso (modo significandi) frente a un intellectus sedundus cuyo significado viene dado no por sí mismo, sino solo en función de la intención de significar particular del emisor (intentio proferentis $)^{34}$. El éxito del discurso figurado hallaba de este modo justificación, pero,

31 La exigencia de inteligibilidad lleva a considerar a Pedro Helías enunciados gramaticalmente incorrectos (non congrua uoce), pero comprensibles para el oyente (congrua sensu). Turba ruunt es el ejemplo propuesto por el gramático de Poitiers quien aplica ya la denominación de figura para este tipo de enunciados, (Reilly 1993, 833). En el mismo sentido, pero a la inversa, Helías también reflexiona sobre las oraciones gramaticalmente correctas (congrua uoce), pero ininteligibles (non congrua sensu) mediante el ejemplo Socrates habet ypoteticos sotulares cum cathegoricis corrigiis, (Reilly 1993, 833). En suma, lo que el gramático de Poitiers plantea, por vez primera a mediados del siglo XII, es una problemática tan actual como la de la gramaticalidad frente a la interpretabilidad de un enunciado, debate lingüístico que, más de ochocientos años después, Noam Chomsky ha vuelto a abordar en su célebre y revolucionario Syntactic Structures (1957).

32 Aportamos nuestra propia traducción del texto de Pedro Helías.

33 A pesar del trascendental avance de la teoría sintáctica occidental en la segunda mitad del XII que halla su mejor reflejo en la aparición de los primeros tratados dedicados en su totalidad al análisis sintáctico, no se encuentra en estos primeros manuales independientes de sintaxis desarrollo doctrinal ni teórico relativo a la problemática del discurso figurado.

34 En el comentario al Priscianus Minor titulado Sermocinalis Scientia atribuido a un desconocido «Magister Jordanus» y fechado en torno a 1240 figura una de las primeras referencias conservadas a la teoría del doble intellectus, uid. Sirridge $(1980,66)$. Apenas unos años más tarde, los dos autores más destacados e influyentes de mediados del XIII, Robert Kilwardby y Roger Bacon, recogen también dicha teoría y operan con ella a lo 
al mismo tiempo, dicha explicación rebasaba los límites del discurso gramatical y lingüístico en sentido estricto. Y es en esta contradicción donde radica el antecedente y el marco teórico de la toma de postura modista respecto a los enunciados figurados, pues, a mediados del XIII y a partir de las posibilidades que ofrecía la teoría del doble intellectus, dos son los enfoques claramente enfrentados de estudio y análisis de las construcciones figuradas. Sus principales representantes son también, sin lugar a dudas, las dos figuras más relevantes del denominado premodismo: el franciscano inglés Roger Bacon y el dominico -también inglés-Robert Kilwardby.

Bacon representa la corriente minoritaria en el París de mediados de siglo, la que defiende situar en el centro mismo de la reflexión gramatical el discurso figurado. De hecho, al estudio y análisis de construcciones figuradas y anómalas - con toda la carga de intención que ello conlleva- dedica el teólogo y filósofo inglés la práctica totalidad de su relevante Summa Grammatica fechada en torno al $1245^{35}$. Y es que para el teólogo franciscano el discurso figurado, al implicar un emisor, un oyente y una particular y específica situación comunicativa, resulta más perfecto, más rico y más completo que el propiamente gramatical. Y no por propugnar la necesidad de estudiar ambos tipos de discursos, tanto el común como el figurado, renuncia Bacon a la pretensión de universalidad del lenguaje que cifra en una genuina labor filológica de cotejo y análisis de similitudes esenciales entre lenguas distintas ${ }^{36}$.

Sin embargo, en la Facultad de Artes de la Universidad parisina era otra la corriente de pensamiento lingüístico que por entonces triunfaba y se imponía. Su ideólogo no era otro que Robert Kilwardby, posiblemente, el pensador y erudito más influyente no ya del siglo XIII, sino de la Baja Edad Media en general.

La fundamentación científica y universal de la gramática, apoyada en la física aristotélica, articula siempre la exposición doctrinal del dominico inglés. La presentación más completa a este respecto figura en su obra De Ortu Scientiarum (ca. $1250)^{37}$. Y también se recogen diferentes apuntes y explicaciones de esta equiparación en el Comentario al Priscianus Minor, la principal obra gramatical del filósofo inglés de gran influencia posterior y texto de referencia clave para la siguiente generación de gramáticos modistas ${ }^{38}$.

En la exposición de sus ideas gramaticales Robert Kilwardby dedica amplio espacio y considerable atención al análisis de las figuras de construcción; pero el enfoque y la orientación que el filósofo inglés propugna al respecto es justamente el contrario al de Bacon. En efecto, en su afán de conferir a la gramática el estatus de una ciencia de carácter universal -directriz esencial de toda su obra-Kilwardby choca irremediablemente con una amplia serie de enunciados que no se ajustan a las reglas generales de la gramática. Se trata de enunciados anómalos que abarcan desde los

largo de su análisis gramatical y lingüístico, uid. Kilwardby (SPM, Cambridge 191, ff. 185v-186r) y Kilwardby (SPM, Oxford 119, f. 84v); Steele (1940, 17-27). Cf. Kneepkens (1983, 128).

35 Steele $(1940,1-190)$. En la primera parte de su Summa Grammatica Bacon expone los principios que rigen las construcciones figuradas, (Steele 1940, 1-73); en la segunda el franciscano inglés analiza distintas construcciones anómalas, (Steele 1940, 74-129); y en la tercera discute ejemplos -varios tratados ya previamente- que presentan alguna dificultad sintáctica especial, (Steele 1940, 129-190).

36 Cf. Nolan - Hirsch $(1902,27)$ : grammatica una et eadem est secundum substantiam in omnibus linguis, licet accidentaliter uarietur.

37 Cf. Alessio (2011, 107-135).

38 La falta, no obstante, de una edición definitiva del Comentario al Priscianus Minor de R. Kilwardby dificulta en gran medida el análisis exhaustivo y preciso del mismo. 
casos de la licencia poética y crítica literaria hasta aquellos que pueden explicarse mediante el recurso a ciertas reglas particulares de la gramática ${ }^{39}$.

Para lograr justificar el funcionamiento de todos estos enunciados impropios, el filósofo recurre en todos los casos sin excepción a la teoría del doble intellectus ${ }^{40} \mathrm{y}$, más en concreto, a la intención particular del emisor (intentio proferentis) ${ }^{41}$.

Kilwardby conseguía, de este modo, mantener dentro de la gramática -de manera razonada- todos los enunciados que se desviaban de la norma. Pero, al mismo tiempo, aunque no los excluía, sí los apartaba de la gramática general y canónica, la que realmente -según el dominico inglés- debía ser objeto de análisis y consideración por su condición de ciencia y alcance universalista. Y, además, la gramaticalidad que el filósofo inglés confería a los enunciados anómalos resultaba extremadamente limitada, pues dependía tan solo de una intención particular e idiosincrática del emisor ${ }^{42}$.

Por lo tanto, Robert Kilwardby, con su marcada distinción entre el discurso común y el discurso figurado, y la preeminencia absoluta del primero sobre el segundo, establecía ya las bases teóricas que iban a marcar la evolución del pensamiento lingüístico en las décadas siguientes. Y es que de aquí a la definitiva exclusión de la gramática del discurso figurado no había apenas espacio.

Solo faltaba dar un último paso que los modistae dieron con suma facilidad en función de uno de los postulados clave de su teoría, el que establecía el modus significandi como principio único de corrección de todo enunciado. En efecto, según la consideración modista, la corrección del enunciado depende única y exclusivamente de las propiedades intrínsecas de sus constituyentes y no de su uso o de factores extrínsecos, semánticos o subjetivos: ni el emisor ni su intención significativa conciernen ni interesan, por tanto, lo más mínimo al gramático modista.

En suma, los filósofos modistas, con la base doctrinal y teórica del dominico R.

39 De este modo, para R. Kilwardby dos oraciones como Ego et tu disputamus y Urbem quam statuo uestra est responden a un único e idéntico patrón de funcionamiento. Y es que, según la perspectiva teórica del filósofo inglés, todo enunciado figurado constituye una impropiedad producida por una razón (ratio) que tiene, a su vez, un doble aspecto: la razón por la que la impropiedad se debe cometer (ratio qua debet fieri), relacionada con lo que se quiere expresar; y la razón por la que la impropiedad se puede cometer (ratio qua potest fieri), vinculada a las reglas fundamentales de la sintaxis y a las partes del discurso involucradas, uid Kilwardby (SPM, Oxford 119, f. 81r) y (SPM, Cambridge 191, f. 181v): Habito quod sit figura, iam patet ex dictis quaestio secunda, scilicet quid sit; est enim improprietas ratione excusata quia quae non excusatur uitium est. Kilwardby (SPM, Oxford 119, f. 81r) y (SPM, Cambridge 191, f. 181v): Est autem figura constructionis improprietas proueniens in ordinatione constructibilium rationabiliter dicta. Cf. Sirridge (1990, 322-323), Rosier (1998, 198-199).

40 Kilwardby (SPM, Oxford 119, f. 84v) y (SPM, Cambridge 191, f. 185v): Primus intellectus est qui prius cadit in apprehensionem, scilicet qui consistit ex modis significandi dictionum. Secundus est qui secundo comprehenditur, scilicet qui consistit ex significatis dictionum. Cf. Kneepkens (1983, 125-126) y Sirridge (1990, $325)$.

${ }^{41}$ El empeño de R. Kilwardby por explicar y analizar las impropiedades poéticas y literarias como las de carácter gramatical (silepsis, euocatio, etc.) tiene una justificación en el papel que el dominico inglés otorga en su modelo de análisis a la ratio qua debet fieri. Por eso, desarrolla el modelo explicativo de la ratio excusans en el que concede un papel esencial a la ratio qua debet fieri asociada per se a la intentio proferentis; y, por eso, lo aplica a todos los tipos de enunciados anómalos sin excepción, también a aquellos -como los casos de silepsis o euocatio- en los que la alusión a la intención particular del emisor no es en absoluto necesaria para su explicación.

42 El dominico inglés aclara con precisión el tipo de gramaticalidad que corresponde al discurso figurado (oratio figurativa). Kilwardby (SPM, Oxford 119, f. 84v) y (SPM, Cambridge 191, f. 186r): Si quantum ad intellectum significatum, absolute loquendo, talis est oratio incongrua, quamuis rationem habeat et ita secundum quid sit congrua, scilicet secundum intellectum proferentis. Cf. Rosier - Libéra (1986, 71-74), Sirridge (1990, 325-326). 
Kilwardby, rechazan por principio y por sistema la distinción de un doble nivel del lenguaje y el empleo de la figura de construcción, pues operan -al margen del uso y de la semántica- solo en un nivel intrínseco de corrección y gramaticalidad lingüísticas. Y el resultado último de este posicionamiento tan radical será la reducción de la gramática por parte de los modistae a un conjunto de enunciados canónicos alejados por completo de la realidad y riqueza de la comunicación humana que implica siempre y, de manera inevitable, un emisor, un receptor y una singular situación comunicativa:

Expuestos, por tanto, en sus líneas generales los principios básicos del modismo,
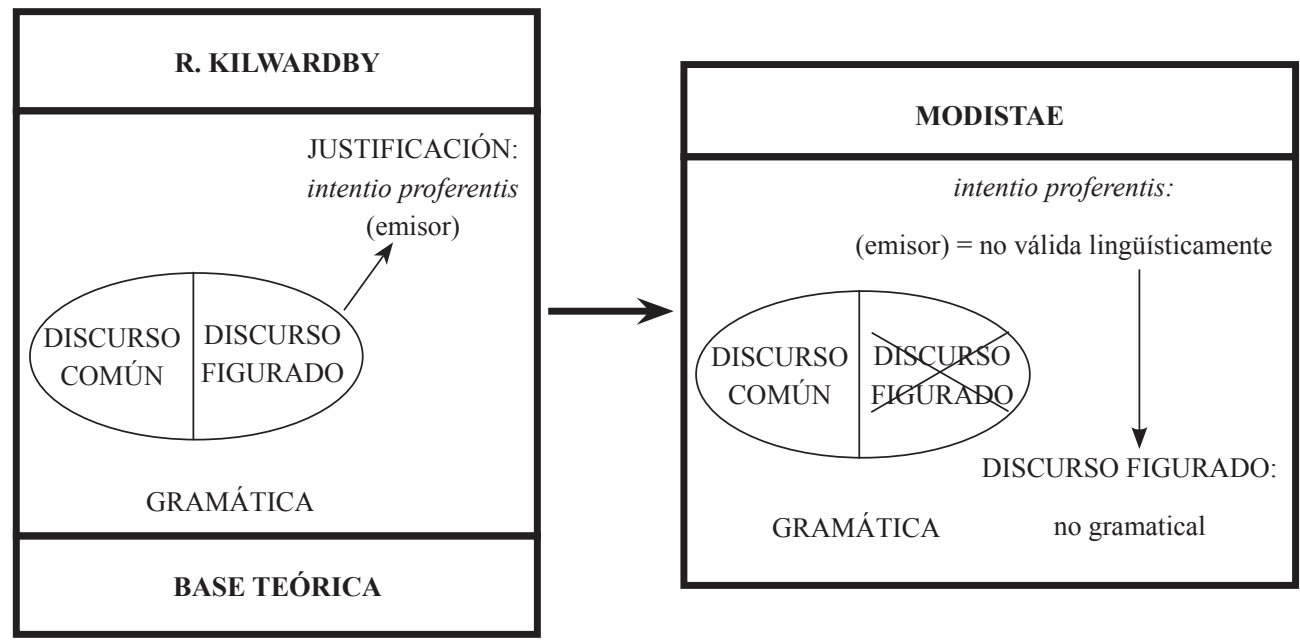

revisemos ahora las marcadas diferencias con la gramática sanctiana que se revelan con total y absoluta evidencia.

\section{La gramática sanctiana: el estudio racional y sistemático del discurso figurado}

En 1587, tras la publicación de la pequeña Minerua, de las Institutiones y de los $P a$ radoxa, Francisco Sánchez de las Brozas publica la Minerua seu de causis linguae latinae, su obra gramatical cumbre y de importancia capital y decisiva en la teoría lingüística de corte racional y teórico posterior ${ }^{43}$.

Y es que desde el inicio mismo de su Minerua el Brocense deja clara -al margen de los propósitos prácticos y didácticos- la perspectiva teórica, general y filosófica que guía, de principio a fin, su obra:

Quae causa fuit ut has nostras uigilias «Mineruam seu de causis linguae latinae» inscriberemus? Quia cuiuslibet quaestionis causas et uera principia eruere conamur [...] (Sánchez Salor - Chaparro Gómez 1995, 42).

¿Por qué llamé Minerva o Causas de la lengua latina a este producto de mis insom-

43 Cf. Breva Claramonte (1980, 351-371), Sánchez Salor (2002, 197-217; 2012, 405-503). 
nios? Porque pretendo descubrir la causa y el verdadero principio de cada cuestión [...] (Sánchez Salor - Chaparro Gómez 1995, 43).

La finalidad última es, por tanto, investigar las causas y fundamentos generales de la lengua latina, yendo más allá de la pura linealidad y concreción material del texto. Se trata, en suma, de elaborar una gramática de carácter universal. Y es que, en el siglo XVI el Brocense -como los modistae tres siglos antes-persigue también un mismo objetivo de universalidad lingüística; pero, a diferencia de los filósofos cultivadores del género de modis significandi, el gramático de las Brozas no excluye las anomalías de uso de su gramática, sino que, muy al contrario -y aquí radica la diferencia esencial-, las convierte en objeto de estudio y análisis sistemático de su obra gramatical.

Y en este sentido, el Brocense otorga a la sintaxis la posición medular de su trata$\mathrm{do}^{44}$. De hecho, de los cuatro libros que conforman la Minerua tres están dedicados al análisis sintáctico.

Libro I: Revisión crítica de las partes orationis.

Libro II: Constructio del nombre

Libro III: Constructio del verbo.

Libro IV: Sintaxis figurada

Pero lo que el gramático de las Brozas estudia y analiza en esos tres libros son construcciones del uso de la lengua latina, es decir -y en marcada contraposición con la teoría sintáctica modista-, construcciones anómalas e imperfectas. A partir de ahí, el modo de proceder del Brocense a lo largo de toda su obra consiste en explicar y analizar dichas anomalías de uso (usus) desde una perspectiva racional y teórica ( $\mathrm{ra}$ tio) en función del principio metodológico básico y medular que vertebra su teoría: la distinción de dos niveles de descripción lingüística ${ }^{45}$.

Y es que en la teoría gramatical sanctiana, como se sabe, juega un papel metodológico esencial la distinción entre un nivel de estructura profunda y racional de reglas abstractas y teóricas, y un nivel de estructura superficial o de realización sintáctica que los modistae, en cambio, como se ha señalado a lo largo de este estudio, rechazan por principio y por sistema.

Asimismo, la figura de construcción que los modistae sitúan fuera de los límites de la gramática adquiere, por el contrario, importancia capital y decisiva en la metodología sanctiana como un mecanismo propiamente metalingüístico que permite explicar los desajustes que se producen entre el nivel de ratio y el de usus.

De las cuatro figuras que el Brocense acepta, siguiendo, en líneas generales, el sistema cuatripartito introducido por Linacro ${ }^{46}$-elipsis, pleonasmo, silepsis e hipérbaton $^{47}$ - la preeminencia de la elipsis resulta evidente. De hecho, la elipsis constitu-

44 Desde el inicio mismo de su Minerua el Brocense identifica syntaxis y oratio que para el humanista extremeño constituyen la finalidad del análisis gramatical, Sánchez Salor - Chaparro Gómez (1995, 46): Sed oratio siue syntaxis est finis grammaticae.

45 Cf. Codoñer Merino (1988, 177-182), Mañas Núñez (1994, 75-85), Sánchez Salor (1996, 39-48; 1998, 748-749; 2002, 493-519).

46 Vid. Harto Trujillo (1998, 468).

47 Sánchez Salor - Chaparro Gómez (1995, 438). 
ye la base y el eje medular de la doctrina gramatical sanctiana, pues, en función de la teoría de la elipsis, el humanista extremeño explica, de manera sistemática, múltiples anomalías de uso respecto a la ratio gramatical ${ }^{48}$. Entre las más importantes cabe señalar la elipsis de un acusativo de la misma raíz que el verbo en los considerados por la tradición como uerba neutra ${ }^{49}$; la omisión de un nominatiuus cognatus en los comúnmente denominados verba impersonalia ${ }^{50}$; la supresión de un sustantivo siempre que un genitivo o un adjetivo aparecen $\operatorname{solos}^{51}$; o el sobreentendimiento de los pronombres personales ego, tu, nos y uos en las primeras y segundas personas de los verbos ${ }^{52}$.

Y este último ejemplo resulta especialmente significativo como reflejo de la contrapuesta perspectiva metodológica del Brocense y los modistae frente al análisis sintáctico. Y es que, mientras que para los cultivadores del género de modis significandi la equivalencia entre dos enunciados como ego lego y lego es -según se ha visto - total y absoluta, para el Brocense, en cambio, en función de su diferente patrón metodológico, la supresión o elipsis de cualquier palabra a nivel formal implica siempre y necesariamente un cambio a nivel semántico. De este modo, para el gramático de las Brozas, dado que el verbo ya indica por sí mismo la categoría de persona, la presencia del pronombre en nominativo (ego) expresa siempre un valor semántico adicional de oposición, énfasis, etc ${ }^{53}$ :

En suma, en la Minerua del Brocense también se opera con reglas generales y

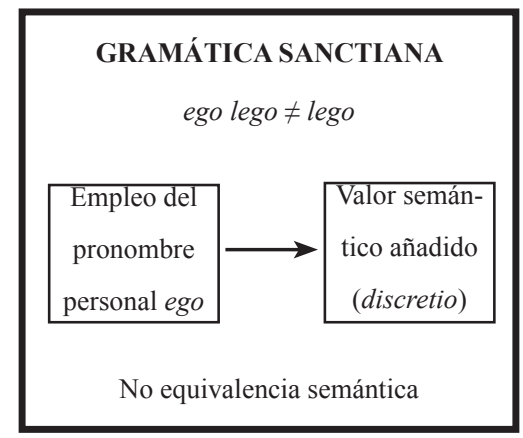

reglas universales de funcionamiento gramatical, pero aplicadas -en radical oposición a lo que sucede en la sistematización modista- a las anomalías de uso a fin de analizarlas y explicarlas de manera racional y teórica.

\section{Conclusión}

A partir del análisis comparativo de la gramática modista y de la gramática sanctiana

\footnotetext{
48 Cf. Dillinger (1974, 184-207), Clerico (1983, 43-56), Hernández Terrés (1984, 13-67), Colombat (1993, 218229), Sánchez Salor (2002, 569-579).

49 Sánchez Salor - Chaparro Gómez (1995, 240, 444).

50 Sánchez Salor - Chaparro Gómez (1995, 226-232, 442-444).

51 Sánchez Salor - Chaparro Gómez (1995, 72, 128, 130, 132, 134, 136, 164, 190, 446, 448, 456).

52 Sánchez Salor - Chaparro Gómez $(1995,450)$.

53 Sánchez Salor - Chaparro Gómez (1995, 450).
} 
desarrollado a lo largo de esta investigación se comprueba con claridad - a pesar de la confusión general que existe en torno a esta cuestión-que los dos principios metodológicos clave sobre los que se asienta la doctrina sanctiana, es decir, la distinción de dos niveles de descripción gramatical y el recurso a la figura de construcción como un mecanismo de explicación lingüística, son rechazados por principio y por sistema en el modelo teórico modista.

Y es que el modismo - con la referencia doctrinal, como se ha comprobado, del premodista y dominico inglés Robert Kilwardby - asienta la finalidad científica y universal de su teoría en una lengua general, abstracta y canónica, alejada de su uso y también, en consecuencia, de la riqueza comunicativa propia, característica y consustancial a toda lengua.

La Minerua del Brocense no supone, por tanto, contrariamente a lo que con frecuencia se afirma, un retorno a posicionamientos modistas, pues el gramático extremeño fundamenta por vez primera la intención universalista en el lenguaje en la práctica y en situación, es decir, en la propia realidad comunicativa y no fuera de ella como lo habían hecho los estudiosos del modus significandi. Al proceder de este modo, el gramático de las Brozas dio un paso absolutamente decisivo en el estudio de los universales lingüísticos de cuya importancia da muestra su notabilísima influencia y trascendencia posterior.

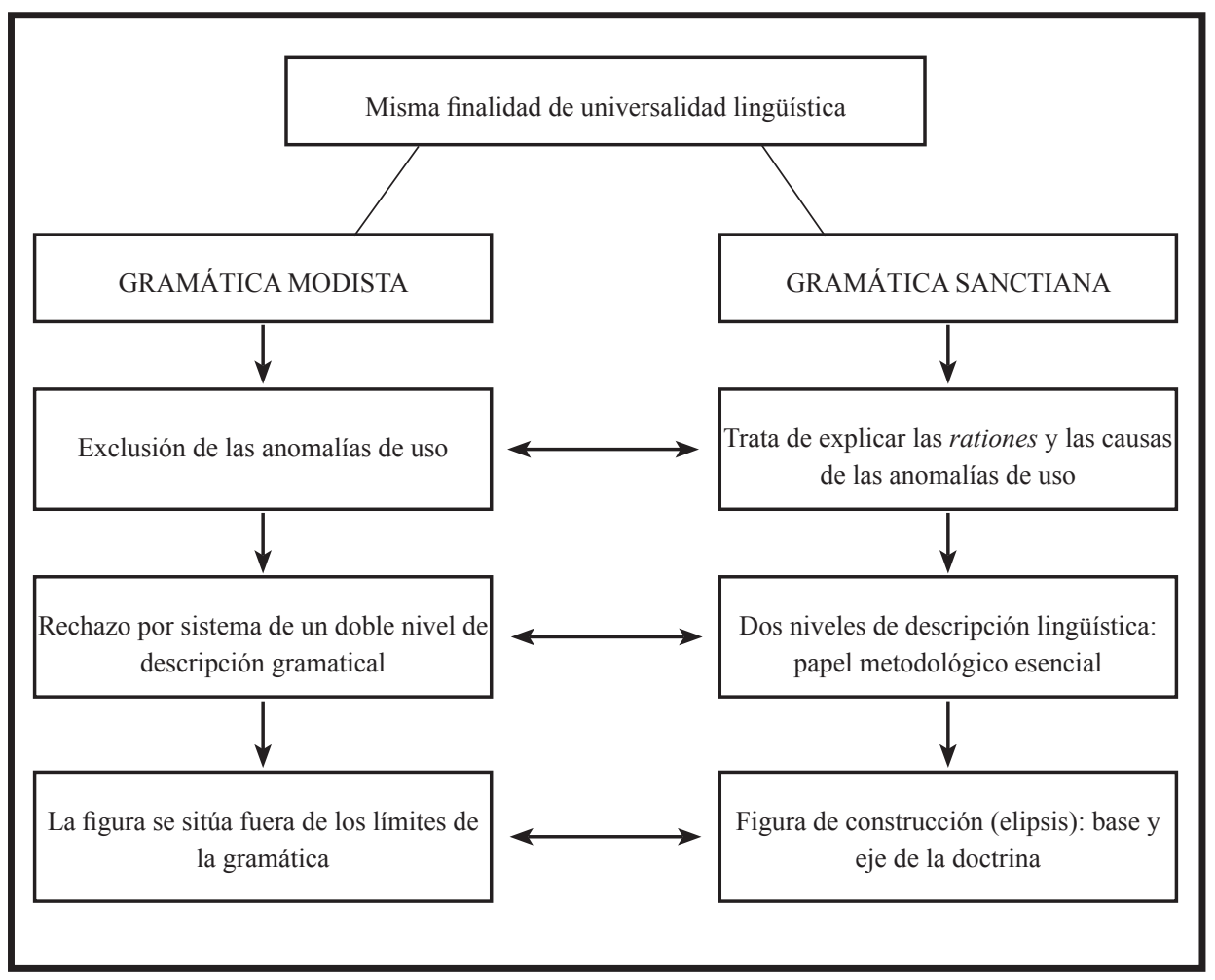




\section{Referencias bibliográficas}

Alessio G.C. (2001), «Sul De ortu scientiarum di Robert Kilwardby», en D’Onofrio, G. (ed.), La divisione della filosofia e le sue ragioni: Lettura di testi medieval, VI-XIII secolo, Salerno, Avagliano Editore, 107-135.

Beuerle, A. (2010), Sprachdenken in Mittelalter: Ein Vergleich mit der Moderne, Berlín Nueva York, Walter de Gruyter.

Breva Claramonte, M. (1980), «La teoría gramatical del Broncese en los siglos XVII y XVIII», RSEL 10.2, 351-371.

-, (1983), Sanctius' Theory of Language. A contribution to the history of Renaissance linguistics, Ámsterdam - Filadelfia, John Benjamins.

Bursill-Hall, G.L. (1971), Speculative Grammars of the Middle Ages. The Doctrine of «Partes orationis» of the Modistae, La Haya - París, Mouton.

-, (1972), Thomas of Erfurt. Grammatica Specultiva. An edition with translation and commentary, Londres, Longman.

-, (1976), «Some Notes on the Grammatical Theory of Boethius of Dacia», en Parret, H. (ed.) History of Linguistic Though and Contemporary Linguistics, Berlín - Nueva York, Walter de Gruyter, 164-188.

Chomsky, N. (1957), Syntatic Structures, La Haya, Mouton.

-, (1966), Cartesian Linguistics: A chapter in the History of rationalist Thought, Nueva York, Harper \& Row.

-, (1968), Language and Mind, Nueva York, Harcourt, Brace \& World.

Clerico, G. (1977), «F. Sanctius: histoire d'une réhabilitation», en Joly, A. - Stéfanini, J. (eds.), La grammaire générale des modistes aux idéologues, Lille, Publications de l'Université de Lille-III, 125-143.

-, (1983), «Ellipse et syntaxe de concordance chez quelques grammairiens classiques», HEL 5.1, 43-56.

Codoñer Merino, C. (1988), «Ratio en el Broncese», en Codoñer Merino, C.; Fernández Álvarez M.P.; Fernández Delgado J.A. (eds.), Stephanion. Homenaje a María C. Giner, Salamanca, Universidad de Salamanca, 177-182.

Colombat, B (1993), Les figures de construction dans la syntaxe latine (1500-1780), Lovaina - París, Peeters.

Covington, M.A. (1984), Syntactic Theory in the High Middle Ages. Modistic Models of Sentence Structure, Cambridge, Cambridge University Press.

Dillinger, D. (1974), History of Linguistics: the Reinassance and Ellipsis in the Grammars of Despauterius, Linacre, Scaliger and Sanctius, Indiana, Indiana University.

Enders, H.W. - Pinborg, J. (1980), Radulphus Brito. Quaestiones super Priscianum Minorem, 2 vols., Stuttgart, Frommann-Halzboog.

Harto Trujillo, M.L. (1998), Thomas Linacer. De emendata structura latini sermonis libri sex, Cáceres, Universidad de Extremadura.

-, (2015), Prisciano. Sintaxis (Sobre la construcción y el orden de las partes de la oración), Madrid, Ediciones Clásicas.

Hernández Terrés, J.M. (1984), La elipsis en la teoría gramatical, Murcia, Universidad de Murcia.

Hertz, M. (1961), Prisciani Grammatici Caesariensis Institutionum Grammaticarum Libri XVIII, GLK II-III, Hildesheim, Georg Olms Verlagsbuchandlung.

Kelly, L.G. (1977), «La Physique d'Aristote et la phrase simple dans les ouvrages de grammaire spéculative», en Joly, A - Stéfanini, J. (eds.), La grammaire générale des modistes aux idéologues, Lille, Publications de l’Université de Lille-III, 107-124. 
-, (2002), The Mirror of Grammar. Theology, philosophy and the Modistae, Ámsterdam Filadelfia, John Benjamins.

Kilwardby, R. (SPM, Cambridge 191), Super Priscianum Minorem, Cambridge Peterhouse 191, ff. 112-229.

—, (SPM, Oxford 119), Super Priscianum Minorem, Oxford Corpus Christi 119, ff. 11-124.

Kneepkens, C.H. (1983), «Roger Bacon's Theory of the Double Intellectus: A note on the

Development of the Theory of Congruitas and Perfectio in the First Half of the Thirteenth Century», en Lewry, P.O. (ed.), The Rise of British Logic: Acts of the Sixth European Symposium on Medieval Logic and Semantics, Toronto, Pontifical Institute of Medieval Studies, 115-143.

Lakoff, G. (1973), «Deep language» [February 8, 1973], The New York Review of Books.

Lakoff, R. (1969), «Review of Grammaire générale et raisonnée, ou La grammaire du Port Royal (by C. Lancelot \& A. Arnauld), ed. Herbert H. Brekle, 2 vols, Stuttgart; Bad Connstatt, Friedick Fromann Verlag, 1966», Language 45, 343-364.

Mañas Nuñez, M. (1994), «El racionalismo del Brocense», Revista de estudios extremeños $50,75-85$.

- (2010), «La teoría gramatical de Sánchez de las Brozas», en González Carrillo, A.M (ed.), «Post tenebras spero lucem». Los estudios gramaticales en la Epaña medieval y renacentista, Granada, Universidad de Granada, 209-235.

Nolan, E - Hirsch, S.A. (1902), The Greek Grammar of Roger Bacon and a Fragment of this Hebrew Grammar. Edited from the mss with introduction and notes, Cambridge, Cambridge University Press.

Pinborg, J - Roos, H. (1969), Boethii Daci opera, CPhD IV, Copenhague, GAD.

Pinborg, J. (1967), Die Entwicklung der Sprachtheorie im Mittelalter, Múnich - Copenhague, Aschendorffsche Verlagsbuchlandlung.

-, (1977), Sigerus de Cortraco. Summa modorum significandi. Sophismata, Ámsterdam, John Benjamins.

Reilly, L. (1993), Pertrus Helias. Summa super Priscianum, 2 vols., Toronto, PIMS.

Rosier, I (1983a), La grammaire spéculative des Modistes, Lille, Presses Universitaires de Lille.

—, (1983b), «Roger Bacon et le problème du sujet sous-entendu», HEL 5.1, 31-42.

-, (1981), «La notion de partie du discours dans la grammaire spéculative», HEL 3.1, 49-62.

- , (1988), «Le traitement spéculatif des constructions figurées au XIII ${ }^{\mathrm{e}}$ siècle», en Rosier, I (ed.), L'héritage des grammariens latins, de l'Antiquité aux Lumières. Actes du Colloque de Chantilly, 2-4 septembre 1987, Lovaina - París, Bibliothèque de l'information grammaticale XIII, 181-204.

Rosier, I. - Libéra, A. (1986), «Intention de signifier et engendrement du discours chez Roger Bacon», HEL 8.2, 63-79.

Roos, H (1961), Martini de Dacia opera, CPhD II, Copenhague, GAD.

Sánchez Salor, E. - Chaparro Gómez, C. (1995), Francisco Sánchez de las Brozas Minerua o De causis linguae latinae, Cáceres, Institución Cultural El Brocense.

-, (1998), «Syntaxis propia y syntaxis figurata en la tradición gramatical», en Nieto Ballester, E. - López Gregoris, R. - Torrego M.E. - García. Hernández, B. (eds.), Estudios de lingüística latina: Actas del IX Coloquio Internacional de Lingüística Latina, vol. 2, Madrid, Ediciones Clásicas, 743-754.

-, (2000), «El Brocense hoy», Alcántara 51, 13-42.

—, (2002), De las «elegancias» a las «causas» de la lengua: retórica y gramática del Humanismo, Alcañiz - Madrid, Instituto de Estudios Humanísticos. 
—, (2003), «La teoría gramatical del Brocense: la oración», en Codoñer Merino, C. - López Moreda, S. - Ureña Bracero, J. (eds.), El Brocense y las humanidades en el s. XVI, Cáceres; Salamanca, Universidad de Salamanca, 61-80.

-, (2012), La gramática en Europa durante el siglo XVII. Dispersión doctrinal, Alcañiz; Madrid, Instituto de Estudios Humanísticos.

Sirridge, M. (1990), «Robert Kilwardby: Figurative Constructions and the Limits of Grammar», en Bursill-Hall, G.L - Ebbensen, S. - Koerner, K. (eds.), De Ortu Grammaticae, Studies in Medieval Grammar and Linguistic Theory in Memory of Jan Pinborg, Ámsterdam; Filadelfia, John Benjamins, 321-337.

-, (1980), «Notulae super Priscianum Minorem Magistri Jordani. Partial edition and introduction», CIMAGL 36, 1-104.

Steele, R. (1940), Summa Grammatica Magistri Rogeri Bacon; Necnon, Sumule Dielactices Magistri Rogeri Bacon, Londres, Oxinii: e Typographeo Clarendoniano.

Stéfanini, J. (1994a), «Une étape dans l'histoire de la linguistique: la Minerva de Sanctius», en Histoire de la grammaire (Textes de J. Stéfanini: réunis par V. Xatard), París, CNRS, 97-107.

—, (1994b), «Les modistes et leur apport à la theorie de la grammaire et du signe linguistique», en Histoire de la grammaire (Textes de J. Stéfanini réunis par V. Xatard), París, CNRS, 53-64.

Thurot, Ch. (1964), Extraits de divers manuscrits latins pour servir à l'histoire des doctrines grammaticales au Moyen Age, Frankfurt, Minerva (reprod. facs. de la ed. de París 1869). 